\title{
Brain white matter oedema due to CIC-2 chloride channel deficiency: an observational analytical study
}

\author{
Christel Depienne*, Marianna Bugiani*, Céline Dupuits, Damien Galanaud, Valérie Touitou, Nienke Postma, Carola van Berkel, Emiel Polder, \\ Eleonore Tollard, Frédéric Darios, Alexis Brice, Christine E de Die-Smulders, Johannes S Vles, Adeline Vanderver, Graziella Uziel, Cengiz Yalcinkaya, \\ Suzanna G Frints, Vera M Kalschever, Jan Klooster, Maarten Kamermans, Truus E M Abbink, Nicole I Wolf, Frédéric Sedelt, Marjo S van der Knaap†
}

\section{Summary}

Background Mutant mouse models suggest that the chloride channel ClC-2 has functions in ion and water homoeostasis, but this has not been confirmed in human beings. We aimed to define novel disorders characterised by distinct patterns of MRI abnormalities in patients with leukoencephalopathies of unknown origin, and to identify the genes mutated in these disorders. We were specifically interested in leukoencephalopathies characterised by white matter oedema, suggesting a defect in ion and water homoeostasis.

Methods In this observational analytical study, we recruited patients with leukoencephalopathies characterised by MRI signal abnormalities in the posterior limbs of the internal capsules, midbrain cerebral peduncles, and middle cerebellar peduncles from our databases of patients with leukoencephalopathies of unknown origin. We used exome sequencing to identify the gene involved. We screened the candidate gene in additional patients by Sanger sequencing and mRNA analysis, and investigated the functional effects of the mutations. We assessed the localisation of ClC-2 with immunohistochemistry and electron microscopy in post-mortem human brains of individuals without neurological disorders.

Findings Seven patients met our inclusion criteria, three with adult-onset disease and four with childhood-onset disease. We identified homozygous or compound-heterozygous mutations in CLCN2 in three adult and three paediatric patients. We found evidence that the CLCN2 mutations result in loss of function of ClC-2. The remaining paediatric patient had an X-linked family history and a mutation in GJB1, encoding connexin 32. Clinical features were variable and included cerebellar ataxia, spasticity, chorioretinopathy with visual field defects, optic neuropathy, cognitive defects, and headaches. MRI showed restricted diffusion suggesting myelin vacuolation that was confined to the specified white matter structures in adult patients, and more diffusely involved the brain white matter in paediatric patients. We detected ClC-2 in all components of the panglial syncytium, enriched in astrocytic endfeet at the perivascular basal lamina, in the glia limitans, and in ependymal cells.

Interpretation Our observations substantiate the concept that ClC-2 is involved in brain ion and water homoeostasis. Autosomal-recessive CLCN2 mutations cause a leukoencephalopathy that belongs to an emerging group of disorders affecting brain ion and water homoeostasis and characterised by intramyelinic oedema.

Funding European Leukodystrophies Association, INSERM and Assistance Publique-Hôpitaux de Paris, Dutch Organisation for Scientific Research (ZonMw), E-Rare, Hersenstichting, Optimix Foundation for Scientific Research, Myelin Disorders Bioregistry Project, National Institute of Neurological Disorders and Stroke, and Genetic and Epigenetic Networks in Cognitive Dysfunction (GENCODYS) Project (funded by the European Union Framework Programme 7).

\section{Introduction}

ClC-2 is a chloride channel that is almost ubiquitously expressed in the human body. ${ }^{1}$ It is present in plasma membranes and is activated by hyperpolarisation, acidic extracellular $\mathrm{pH}$, and osmotic cell swelling. ${ }^{1-4}$ Its functions in human physiology are a matter of debate. Roles in gastric acid secretion, lung development and function, and nephronogenesis have been suggested, but not confirmed. ${ }^{1,5}$ Information about the localisation of $\mathrm{ClC}-2$ within the brain comes from rodent studies, which have detected $\mathrm{ClC}-2$ in oligodendrocytes, astrocytes (especially in endfeet), and pyramidal and non-pyramidal neurons in the hippocampus. ${ }^{6,7}$ Evidence suggests that ClC-2 modulates postsynaptic responses to GABA by affecting intracellular chloride concentration in neurons ${ }^{8}$ and that $\mathrm{ClC}-2$ regulates neuronal excitability, ${ }^{9,10}$ but the physiological relevance of these findings has not been confirmed.

Physiological roles of proteins can be gleaned from diseases caused by mutations in their genes. No human disease has been unequivocally related to mutations in CLCN2, the gene encoding ClC-2. Heterozygous mutations in CLCN2 were described as a cause of idiopathic generalised epilepsies, ${ }^{11-17}$ but this finding was later refuted ${ }^{1,18}$ and the original paper was retracted. ${ }^{17}$ Because mutant mice without functional $\mathrm{ClC}-2$ have a leukoencephalopathy with intramyelinic oedema, ${ }^{5,6,19}$ ClC-2 was proposed to have a role in brain ion and water
Lancet Neurol 2013; 12: 659-68 Published Online May 22, 2013 http://dx.doi.org/10.1016/ S1474-4422(13)70053-X

See Comment page 626

*Authors contributed equally †Joint last authors INSERM, U975-CRICM (C Depienne PhD, F Darios PhD, Prof A Brice MD), AP-HP, Département de Génétique et de Cytogénétique (C Depienne, Prof A Brice), AP-HP, Unité Fonctionnelle Neurométabolique

(CDepienne, CDupuits MSC, F Sedel MD), AP-HP, Service de Neuroradiologie

(Prof D Galanaud MD), AP-HP, Service d'Ophthalmologie (V Touitou), and AP-HP, Département de Neurologie (F Sedel), Hôpital Pitié-Salpêtrière, Paris, France; Université Pierre et Marie Curie-Paris-6 (UPMC), Paris, France (C Depienne, Prof D Galanaud, F Darios, Prof A Brice, F Sedel); Department of Pathology (M Bugiani MD), and Department of Child Neurology (M Bugiani, N Postma, C van Berkel, E Polder, TE M Abbink PhD, N I Wolf MD, Prof M S van der Knaap MD), Neuroscience Campus Amsterdam, VU University Medical Center, Amsterdam, Netherlands; CHU de Rouen, Service de Radiologie, Rouen, France (ETollard MD); Department of Clinical Genetics (Prof C E de Die-Smulders MD, SG Frints MD), and Department of Child Neurology (Prof J S Vles MD), Maastricht University Medical Center, Maastricht, Netherlands; Department of Neurology, Children's National Medical Center, Washington, DC, USA (A Vanderver MD); Unit of Child Neurology, "Carlo Besta" Neurological Institute Foundation, Milan, Italy (G Uziel MD); Division of Child 
Neurology, Department of Neurology, Cerrahpasa Medical School, Istanbul University, Istanbul, Turkey (Prof C Yalcinkaya MD); Department of Human Molecular Genetics, Max Planck Institute for Molecular Genetics, Berlin, Germany (V M Kalscheuer PhD); Department of Retinal Signal Processing, Netherlands Institute for NeuroscienceKNAW, Amsterdam, Netherlands (J Klooster MSc, Prof M Kamermans PhD); and Department of Neurogenetics, Academic Medical Center, Amsterdam, Netherlands (Prof M Kamermans) Correspondence to: Dr Christel Depienne, INSERM, U975-CRICM, Hôpital PitiéSalpêtrière, 75013 Paris, France christel.depienne@upmc.fr

Prof Marjo S van der Knaap, Department of Child Neurology,

VU University Medical Center, 1081 HV Amsterdam Netherlands

ms.vanderknaap@vumc.nl

( images of patients with leukoencephalopathies of unknown origin submitted from all over the world. Patients with prominent signal abnormalities in the middle cerebellar peduncles, midbrain cerebral peduncles, and posterior limbs of the internal capsules were included in the study.

We studied the MRI results of each patient and scored the appearance and distribution of signal abnormalities. We assessed apparent diffusion coefficient (ADC) values in areas of abnormal signal. Signal intensity on $\mathrm{T}_{2}$-weighted and $\mathrm{T}_{1}$-weighted images and $\mathrm{ADC}$ values provide information on tissue microstructure. High ADC values indicate large water spaces; low ADC values indicate small water spaces. Because the study was multi-institutional, MRIs were done with different pulse sequences on machines from different vendors. Therefore, we used a robust cutoff ADC value of $60 \times 10^{-5} \mathrm{~mm}^{2} / \mathrm{s}$, below which the diffusion was considered to be restricted. Subtle diffusion restriction could, therefore, have been missed.

We obtained approval from the institutional review boards of the participating centres in Paris and Amsterdam (Hôpital Pitié-Salpêtrière and VU University Medical Centre) and informed consent from patients and their families.

\section{Procedures}

Exome sequencing was undertaken in the three patients with adult-onset disease under the assumption that these patients had one disease with autosomal recessive inheritance. In patient 3 , who has consanguineous parents, we identified homozygous regions (appendix), in which we searched for homozygous mutations. One paediatric patient came from a family with X-linked disease inheritance, and this family participated in another exome sequencing study focused on X-linked disorders. We then used Sanger sequencing to sequence the CLCN2 gene in both the adult and paediatric patients; GJB1, which encodes the gap junction beta-1 protein (also known as connexin 32), was sequenced in the male patient without CLCN2 mutations (appendix).

CLCN2 mRNA was quantified in fibroblasts of adult patients with CLCN2 mutations. Transient transfections of wild-type or mutant ClC-2-V5-His6 expression plasmids in COS7 cells were used to study the functional effects of CLCN2 mutations (appendix).

To define which cell types express ClC-2 in healthy mature human brain, we examined the posterior limb of the internal capsule, frontal white matter, frontal cortex, and ependyma with fluorescence immunohistochemistry of tissue obtained at autopsy from nine patients aged 1-75 years (appendix) who succumbed to heart disease or extracerebral neoplasm. None of the patients had neurological symptoms and neuropathological examination showed no abnormalities.

Immunohistochemistry (appendix) was done on frozen tissue using antibodies against ClC-2, GlialCAM, MLC1, glial fibrillary acidic protein (GFAP; an astrocyte marker), oligodendrocyte transcription factor-2 (OLIG2; an oligodendrocyte marker), neuronal nuclear antigen (NeuN; a neuronal marker, also known as RBFOX3), platelet endothelial cell adhesion molecule-1 (PECAM1; an endothelial marker, also known as CD31), and phosphorylated and non-phosphorylated neurofilament H (SMI31 and SMI32, respectively; axonal markers). The anti-ClC-2 antibodies were validated for specificity (appendix). All sections were counterstained with DAPI (4 ,6-diamidino-2-phenylindole; nuclear staining).

Electron microscopy was done on tissue from the posterior limb of the internal capsule (appendix). Sections were incubated with ClC-2, GlialCAM, and MLC1 antibodies.

\section{Role of the funding source}

The sponsors of the study had no role in study design, data collection, data analysis, data interpretation, or writing of the report. The corresponding authors had full access to all the data in the study and had final responsibility for the decision to submit for publication.

\section{Results}

Seven patients met our inclusion criteria, three with adultonset disease and four with childhood-onset disease. The three patients with adult-onset disease were unrelated (patients 1-3; table 1). They had mild cerebellar ataxia and a variable combination of chorioretinopathy with visual field defects, optic neuropathy, and headaches. One patient had a schizophrenia-like disorder. The three patients with childhood-onset disease and CLCN2 
mutations (patients 4-6) had mild cerebellar ataxia and a variable combination of mild spasticity, visual field defects, learning disabilities, and headaches (table 1).

The patient with X-linked disease (patient 7; table 1) presented with nystagmus at 12 months. He developed incapacitating cerebellar ataxia and spasticity. Clinical evidence of peripheral nerve involvement was absent, although recent neurophysiological investigations revealed mild motor neuropathy. Affected male family members had the same clinical and MRI findings. Carrier females had no abnormalities.

All patients had prominent signal abnormalities and decreased ADC values in the posterior limbs of the internal capsules, cerebral peduncles in the midbrain, pyramidal tracts in the pons, and middle cerebellar peduncles (figure 1; appendix). All patients with adultonset disease had additional signal abnormalities in specific brainstem tracts and the cerebellar white matter (appendix). Cerebral hemispheric white matter abnormalities were mild and non-specific. In one patient (patient 1), low to borderline-low ADC values were also found in cerebral white matter areas (appendix).
In the paediatric patients, the same additional signal abnormalities in specific brainstem tracts and cerebellum were noted. Additionally, they had diffuse mild signal abnormality of the brain white matter, which was hyperintense relative to grey matter on $\mathrm{T}_{2}$-weighted and $\mathrm{T}_{1^{-}}$weighted images (figure 1). Low to borderline-low ADC values were present in the cerebral and cerebellar hemispheric white matter and corpus callosum. The signal behaviour of the white matter on $\mathrm{T}_{1}$-weighted and $\mathrm{T}_{2}$ weighted images would typically suggest hypomyelination, ${ }^{29}$ but hypomyelination is associated with increased size of water spaces and therefore increased ADC values. ${ }^{30}$ The low white matter ADC suggests myelin microvacuolation instead of hypomyelination. ${ }^{31}$ Increased intramyelinic water accounts for the mild $\mathrm{T}_{2}$-signal changes.

As a result of exome sequencing and homozygosity mapping in patient 3, we identified ten homozygous single nucleotide substitutions and two homozygous insertion-deletions with a possible effect on gene or protein function that were absent from control databases (appendix). One was a homozygous in-frame deletion (p.Leu144_Ile145del) in CLCN2. Analysis of exome data

\begin{tabular}{|c|c|c|c|c|c|c|c|}
\hline & Patient 1 & Patient 2 & Patient 3 & Patient 4 & Patient 5 & Patient 6 & Patient 7 \\
\hline Year of birth & 1948 & 1952 & 1961 & 1996 & 1998 & 2001 & 1993 \\
\hline Sex/ancestry & f/north Africa & f/north Africa & f/north Africa & f/Europe & $\mathrm{m} /$ Europe & f/Europe & $\mathrm{m} /$ Europe \\
\hline Consanguineous parents & No & No & Yes & Not known & No & Yes & No \\
\hline Affected family members & No & No & No & No & No & No & Yes \\
\hline $\begin{array}{l}\text { Early psychomotor } \\
\text { development }\end{array}$ & Normal & Normal & Normal & Normal & Normal & Normal & $\begin{array}{l}\text { Normal, but has never } \\
\text { walked without support }\end{array}$ \\
\hline Age at first sign & 44 years & 57 years & 30 years & 12 years & 6 years & 3 years & 12 months \\
\hline Presenting signs & $\begin{array}{l}\text { Action tremor, } \\
\text { mild gait ataxia }\end{array}$ & Tinnitus, vertigo & $\begin{array}{l}\text { Chorioretinopathy, } \\
\text { psychosis }\end{array}$ & $\begin{array}{l}\text { Learning disability, } \\
\text { headache }\end{array}$ & Headache & $\begin{array}{l}\text { Action tremor, } \\
\text { mild gait ataxia }\end{array}$ & Nystagmus \\
\hline Disease course & Stable & Progressive & Stable & Stable & Stable & Stable & Slowly progressive \\
\hline Signs of the deterioration & None & Deafness & None & None & None & None & Motor deterioration \\
\hline Headache & No & No & Yes & Yes, severe & Yes, severe & No & No \\
\hline Cognitive level & Normal & Normal & Severe learning disability & $\begin{array}{l}\text { Mild learning } \\
\text { disability }\end{array}$ & Normal & $\begin{array}{l}\text { Mild learning } \\
\text { disability }\end{array}$ & Mild learning disability \\
\hline Head circumference & Normal & Normal & Normal & Normal & Normal & $\begin{array}{l}\text { Borderline } \\
\text { macrocephaly }\end{array}$ & Borderline macrocephaly \\
\hline Vision & Normal & $\begin{array}{l}\text { 20/80 right eye; } \\
20 / 400 \text { left eye }\end{array}$ & $\begin{array}{l}1 / 10 \text { right eye; } 1 / 50 \text { left } \\
\text { eye }\end{array}$ & Normal with glasses & $\begin{array}{l}\text { Normal with glasses, } \\
\text { strabismus }\end{array}$ & Normal & Normal \\
\hline Visual field defects & No & Yes & Yes & Yes & No & Yes & No \\
\hline Retina and optic nerve & Normal & $\begin{array}{l}\text { Retinoschisis, } \\
\text { bilateral optic } \\
\text { neuropathy }\end{array}$ & $\begin{array}{l}\text { Retinal atrophy, choroidal } \\
\text { neovascularisation, } \\
\text { bilateral optic neuropathy }\end{array}$ & Normal & Normal & Normal & Normal \\
\hline Nystagmus & No & No & No & No & Yes & No & Yes \\
\hline Hearing & Normal & $\begin{array}{l}\text { Perceptive } \\
\text { hearing loss }\end{array}$ & Normal & Normal & Normal & Normal & Normal \\
\hline Spasticity & No & No & No & Yes, mild & No & Yes, mild & Yes, prominent \\
\hline Ataxia & Yes, mild & Yes, mild & Yes, mild & Yes, mild & Yes, mild & Yes, mild & Yes, severe \\
\hline Peripheral polyneuropathy & No & No & No & No & No & No & $\begin{array}{l}\text { No clinical signs; mildly } \\
\text { decreased motor NCV }\end{array}$ \\
\hline Gait & Unstable & Unstable & Unstable & Unstable & Normal & Unstable & Wheelchair dependent \\
\hline
\end{tabular}




\section{Articles}

in the other adult patients revealed the same homozygous non-sense mutation (p.Trp570X) in CLCN2 (table 2; appendix). CLCN2 was the only gene that contained at

\section{Adult patient with CLCN2 mutations}
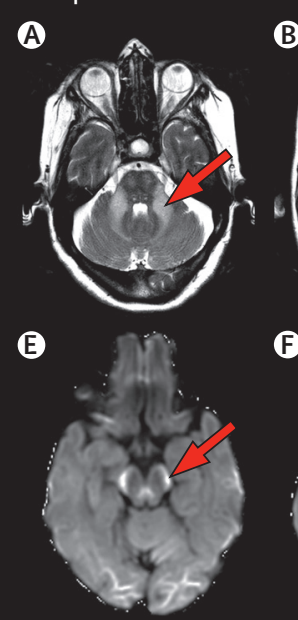

(F)
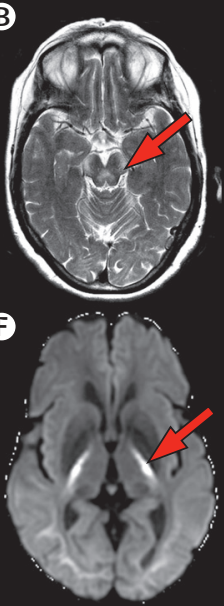

Paediatric patient with CLCN2 mutations
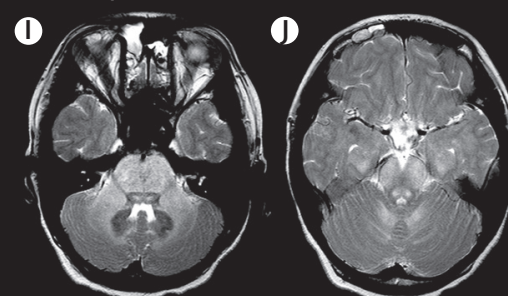

(M)

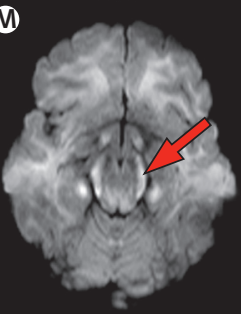

(N)
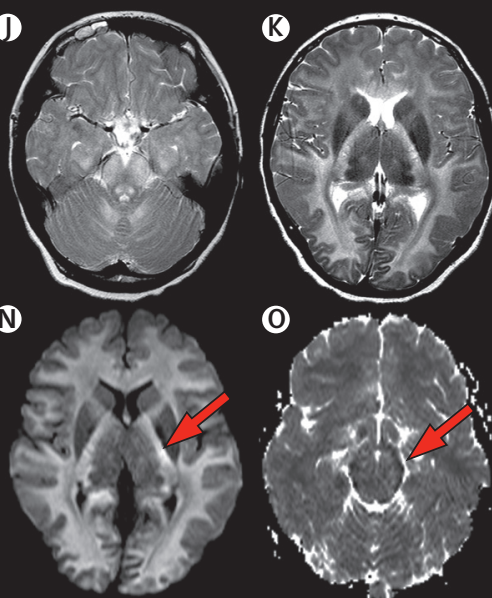

(0)
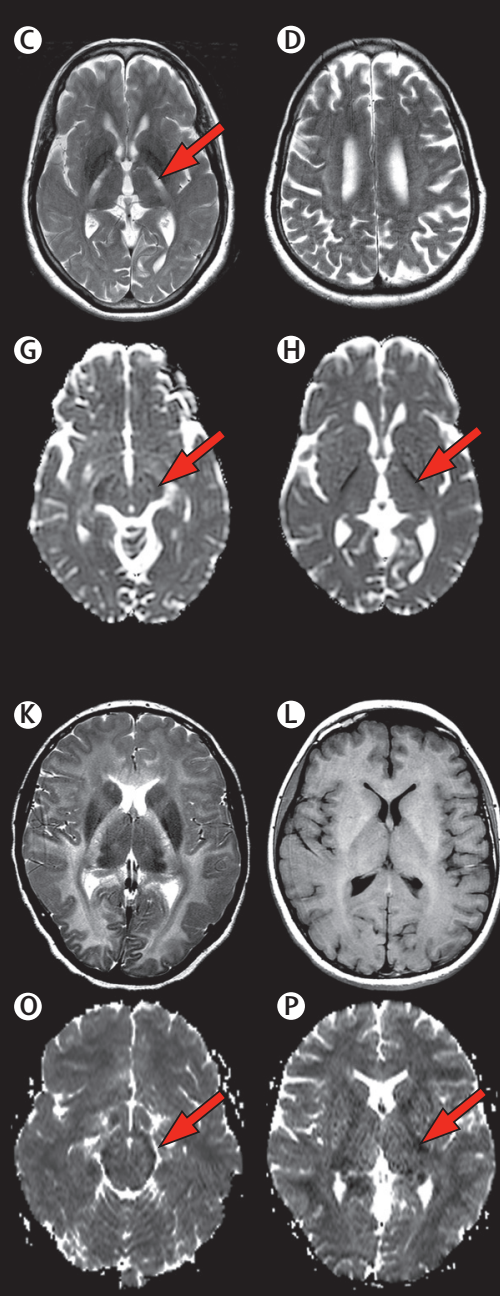

Paediatric patient with GJB1 mutation
Q

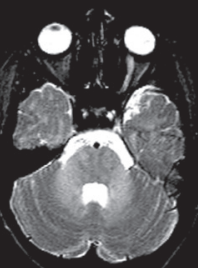

0

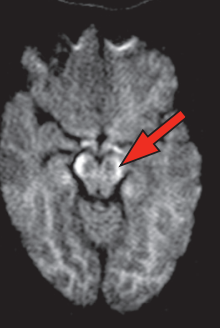

(R)

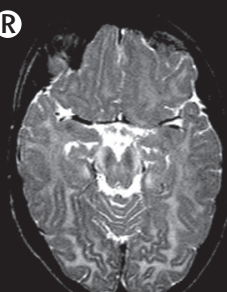

V

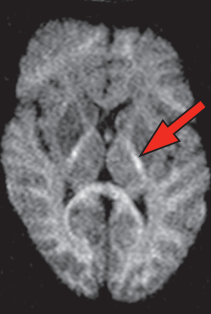

5

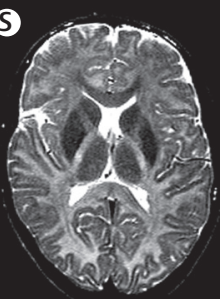

W

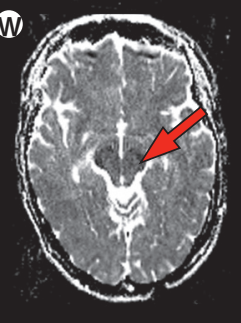

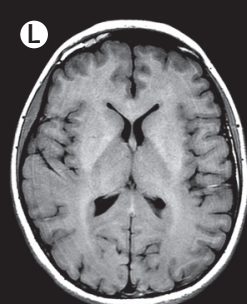

(P)

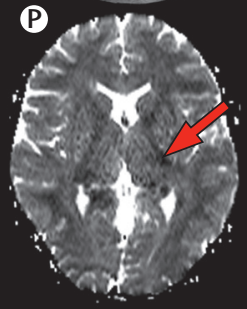

least one homozygous or at least two heterozygous, possibly deleterious variants in all three patients that were absent from exome data from individuals with other diseases analysed at the same time (appendix).

Analysis of the coding sequence of CLCN2 by Sanger sequencing in the paediatric patients identified homozygous or compound-heterozygous CLCN2 mutations in three patients (table 2; appendix). All mutations were predicted to be pathogenic and none was present in healthy controls of the same origin or in the International Hapmap Project and the 1000 Genomes Project databases (appendix).

Sequencing of the exome of the paediatric patient without CLCN2 mutations who had an X-linked family history revealed a novel missense mutation (p.Pro174Ser) in GJB1 (table 2; appendix). This mutation was confirmed in his mother and in one living similarly affected male family member.

Quantification of the CLCN2 mRNA in fibroblasts from patients and controls by real-time quantitative PCR showed that the mRNA with the p.Trp570X mutation was substantially downregulated compared with that of controls, by contrast with the mRNA with p.Leu144_Ile145del (appendix). Treatment with emetine, an inhibitor of non-sense mediated decay, rescued the mRNA expression, suggesting that p.Trp570X leads to degradation of the mutant mRNA, although a substantial fraction of the mRNA subsists. We could not confirm the presence of the truncated protein because expression of ClC-2 was undetectable in fibroblasts. Transient expression of the protein with the p.Trp570X mutation in COS7 cells showed that the truncated protein, if expressed, has an aberrant localisation restricted to the Golgi apparatus (appendix).

\section{Figure 1: MRI of brain in patients 1,4 , and 7}

MRI in patient 1 at the age of 63 years $(A-H)$ shows signal abnormalities on $T_{2}$-weighted images (A-D) in the middle cerebellar peduncles (arrow in $A$ ), midbrain cerebral peduncles (arrow in B), and posterior limbs of the internal capsules (arrow in C). Slight signal abnormalities are present in the cerebral white matter (D). Diffusion-weighted images ( $E, F)$ show high signal in the midbrain cerebral peduncles (arrow in E), posterior limbs of the internal capsules (arrow in F), and to a lesser extent the posterior subcortical cerebral white matter (compare hyperintense posterior white matter to normal anterior white matter in $E$ and $F$ ), suggesting restricted diffusion, confirmed by low $A D C$ values on the $A D C$ maps (low signal in $\mathrm{G}$ and $\mathrm{H}$, arrows). MRI in patient 4 at the age of 14 years (I-P) shows diffuse mild $\mathrm{T}_{2}$-hyperintensity $(\mathrm{I}-\mathrm{K})$ and $\mathrm{T}_{1}$-hyperintensity $(\mathrm{L})$ of all white matter structures. Prominent $\mathrm{T}_{2}$-hyperintensity is seen in the basis pontis (I), middle cerebellar peduncles (I), midbrain cerebral peduncles $(\mathrm{J})$, and posterior limbs of the internal capsules ( $K$ ). Diffusion-weighted images $(M, N)$ show increased signal in virtually all white matter, especially the midbrain cerebral peduncles (arrow in M) and posterior limbs of the internal capsules (arrow in N), suggesting restricted diffusion, confirmed by low $A D C$ values on the $A D C$ maps (arrows in $\mathrm{O}$ and $\mathrm{P}$ ). MRI in patient 7 at the age of 14 years $(\mathrm{Q}-\mathrm{X})$ shows diffuse mild $\mathrm{T}_{2}$-hyperintensity $(\mathrm{Q}-\mathrm{S})$ and $\mathrm{T}_{1}$-hyperintensity $(\mathrm{T})$ of all white matter structures. Prominent $\mathrm{T}_{2}$-hyperintensity is seen in the middle cerebellar peduncles $(Q)$, midbrain cerebral peduncles $(R)$, and posterior limbs of the internal capsules (S). Diffusion-weighted images $(U, V)$ show mildly increased signal in almost all white matter, especially the midbrain cerebral peduncles (arrow in $U$ ) and posterior limbs of the internal capsules (arrow in V), suggesting restricted diffusion, confirmed by low ADC values on the ADC maps (arrows in W and X). 


\begin{tabular}{|c|c|c|c|c|c|c|}
\hline & Exon & DNA & RNA & Protein & Genotype & Confirmation in parents* \\
\hline \multicolumn{7}{|c|}{ CLCN2 mutation } \\
\hline Patient 1 & 15 & c. $709 \mathrm{G} \rightarrow \mathrm{A}$ & .. & p.Trp570X & Homozygous &.. \\
\hline Patient 2 & 15 & c. $1709 \mathrm{G} \rightarrow \mathrm{A}$ & .. & p.Trp570X & Homozygous &.$\cdot$ \\
\hline Patient 3 & 4 & c.430_435del & .. & p.Leu144_lle145del & Homozygous &.. \\
\hline Patient 4 & $11 ; 2$ to part of 6 & $\begin{array}{l}\text { c.1143delT; } \\
\text { c.64-1107_639del }\end{array}$ & $\begin{array}{l}\text { r.1143delt; } \\
\text { r.64_639delins82† }\end{array}$ & $\begin{array}{l}\text { p.Gly382AlafsX34; } \\
\text { p.Met22LeufsX5 }\end{array}$ & $\begin{array}{l}\text { Heterozygous; } \\
\text { heterozygous }\end{array}$ &.. \\
\hline Patient 5 & 14 & c.1499C $\rightarrow T$ & $r .1499 c \rightarrow t$ & p.Ala500Val & Homozygous & Father and mother \\
\hline Patient 6 & 8 & c.828dupG &.. & p.Arg277AlafsX23 & Homozygous & Father and mother \\
\hline \multicolumn{7}{|c|}{ GJB1 mutation } \\
\hline Patient 7 & 2 & c. $520 C \rightarrow T$ &. & p.Pro174Ser & Hemizygous & Mother \\
\hline \multicolumn{7}{|c|}{$\begin{array}{l}\text { *This column shows whether the mutation was confirmed in the father and mother of the affected patient. †Comprises a deletion of exons } 2-5 \text { and part of exon } 6 \text { and the } \\
\text { insertion of intron } 1 \text { c. } 63+1097 \_64-1108 .\end{array}$} \\
\hline
\end{tabular}

The p.Leu144_Ile145del and p.Ala500Val mutations are thought to affect conserved hydrophobic aminoacids located in transmembrane domains of $\mathrm{ClC}-2$, possibly leading to protein misfolding or misinsertion in the membrane. To test this hypothesis, we compared the expression and subcellular localisation of V5-His6-tagged ClC-2 with the p.Leu144_Ile145del or p.Ala500Val mutation to those of the wild-type protein in COS7 cells. Localisation of the p.Leu144_Ile145del ClC-2 and p.Ala500Val ClC-2 proteins was restricted to the endoplasmic reticulum, whereas wild-type ClC-2 was present in different subcellular compartments including the plasma membrane (figure 2A). Additionally, the amount of both mutated $\mathrm{ClC}-2$ proteins transiently expressed in COS7 cells was lower than the amount of wild-type ClC-2 (figure 2B) and the mutated proteins hardly reached the plasma membrane (figure 2C and 2D). These findings suggest that p.Leu144_Ile145del ClC-2 and p.Ala500Val ClC-2 are trapped and degraded in the endoplasmic reticulum. Altogether, these results suggest that all mutations identified in the patients led to a complete or at least partial loss of function of the $\mathrm{ClC}-2$ channel by different mechanisms.

Immunohistochemistry of the healthy brain samples revealed that virtually all GFAP-positive fibrous astrocytes in the posterior limb of the internal capsule and frontal white matter express $\mathrm{ClC}-2$ on the surface of cell bodies and processes (figure $3 \mathrm{~A}$ ). $\mathrm{ClC}-2$ immunopositivity had a fine punctate quality (figure $3 \mathrm{~A}$, inset), as expected for a membrane protein. Many ClC-2-positive astrocytic processes ran parallel and perpendicular to axonal bundles (figure 3A) and abutting axons (appendix). ClC-2 was enriched in perivascular astrocytes (figure 3B), where GlialCAM (figure 3C; appendix) and MLC1 (appendix) were also expressed. GlialCAM and MLC1 immunoreactivity overlapped consistently and extended further distally than ClC-2 into astrocytic endfeet approaching the basal lamina (figure 3C; appendix). A more discrete, punctate $\mathrm{ClC}-2$ immunoreactivity was visible intermittently along axons (appendix). We detected many ClC-2-positive and GFAP-negative cells with the morphology of oligodendrocytes. In the frontal cortex, ClC-2 expression was detected only in scattered protoplasmic astrocytes around blood vessels and in astrocytes that are part of the glia limitans (figure 3D; appendix). In these locations, $\mathrm{ClC}-2$ astrocytes coexpressed GlialCAM (figure 3E; appendix) and MLC1 (appendix). ClC-2 expression was also detected in the ependymal lining, again overlapping with GlialCAM (figure 3F; appendix) and MLC1 (appendix). No ClC-2-positive expression was noted in neuronal perikarya (figure 3G).

Electron microscopy confirmed that $\mathrm{ClC}-2$ was abundantly present in white matter astrocytes and enriched in cell processes contacting abaxonal myelin (figure 4A) and contacting each other (figure 4B). At these sites, GlialCAM showed a similar distribution (figure 4C, and 4D). ClC-2 immunoreactivity was also visible inside axons and at the axonal surface contacting adaxonal myelin (appendix). By contrast, GlialCAM and MLC1 were not detected in axons or myelin (figure 4C). Around blood vessels, ClC-2 was found at astrocyte-astrocyte contacts in endfeet (figure 4B), a site where GlialCAM (figure 4D) and MLC1 (figure 4E) were present.

\section{Discussion}

We showed that autosomal recessive loss-of-function mutations in CLCN2 cause a leukoencephalopathy with MRI evidence of myelin microvacuolation. These findings are similar to those in ClC-2-deficient mice and are in line with the notion that $\mathrm{ClC}-2$ in the brain is involved in ion and water homoeostasis (panel). ${ }^{5,6}$

Our observations do not support the hypothesis that loss-of-function mutations in CLCN2 confer an increased risk of epilepsy. None of the six patients with homozygous or compound heterozygous mutations in CLCN2 had epilepsy or a family history of epilepsy, suggesting that partial or complete $\mathrm{ClC}-2$ loss of function per se is insufficient to cause epilepsy.

The disease associated with CLCN2 mutations is variable in age of onset and clinical presentation. Mild 
cerebellar ataxia is the most consistent finding. Retinopathy, as reported in the ClC-2-deficient mice, was detected in only some of the patients, although the visual field defects in absence of other ophthalmological findings suggest that the other patients might have had a retinopathy that was too subtle to be picked up at funduscopy. ClC-2-deficient mice are sterile because of testicular degeneration; however, the only male patient with CLCN2 mutations (aged 15 years) did not yet show signs of testicular degeneration. Although the clinical presentation of the patients is non-specific and does not allow a diagnosis, MRI findings in these patients are highly specific and do allow a diagnosis. We have also analysed the CLCN2 gene in several other adult and paediatric patients who had similar MRI findings to those of study patients, but did not meet the MRI inclusion criteria, and we did not find CLCN2 mutations in any of them (data not shown).

The phenotypic variability reported in the patients might at least in part be related to the extent of ClC-2 loss of function. Both the mutational variation and functional analyses suggest loss of function of $\mathrm{ClC}-2$, but some

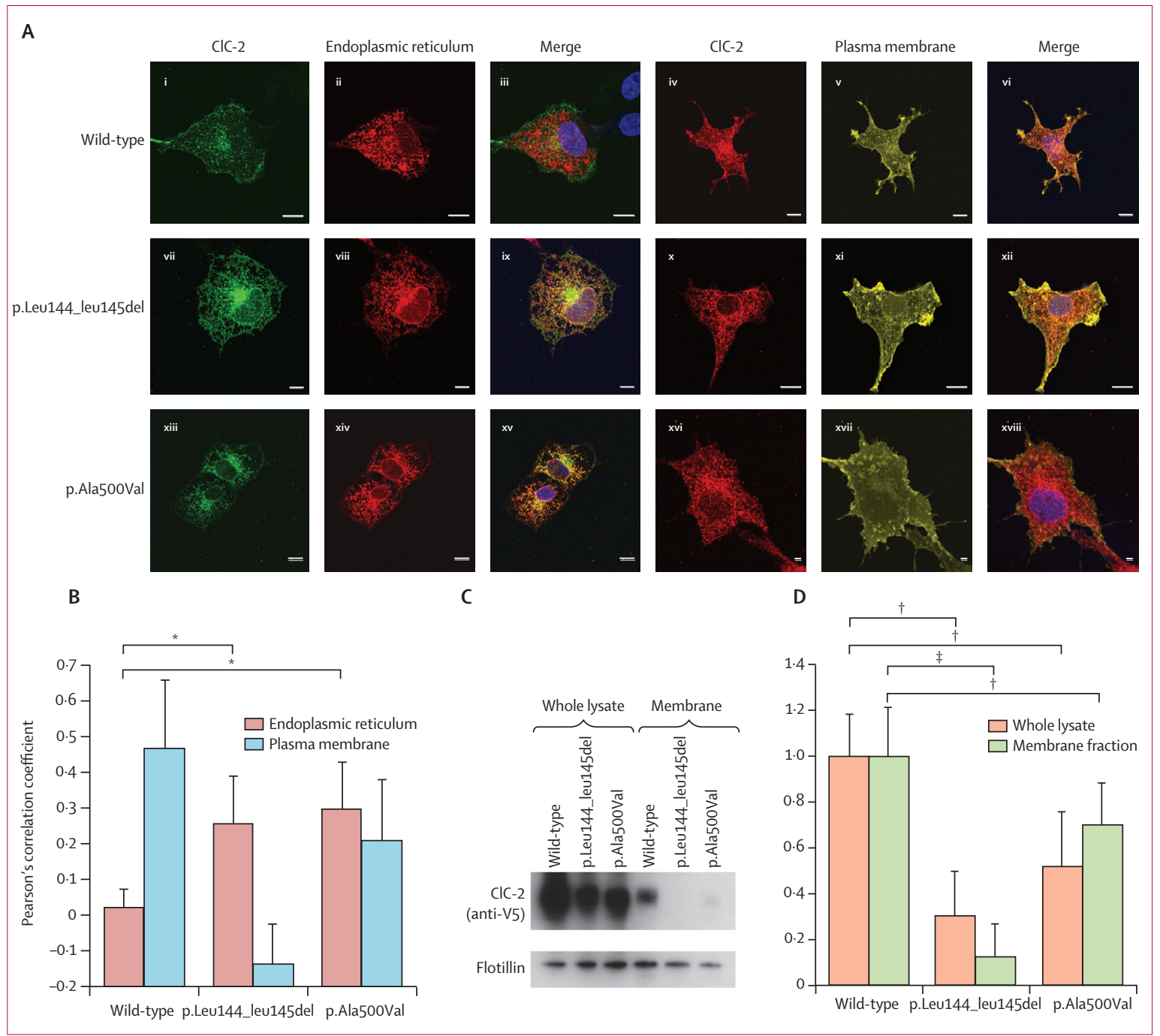

Figure 2: Expression and subcellular localisation of wild-type and mutated CIC-2

(A) Subcellular localisation of wild-type (i-vi) and mutated (p.Leu144_lle145del [vii-xii]; p.Ala500Val [xiii-xviii]) ClC-2 proteins and colocalisation with markers of endoplasmic reticulum (anticalreticulin, red, [ii, viii, xiv]) or plasma membrane (pEYFP-Mem, yellow [v, xi, xvii]) by confocal microscopy. Note that wild-type CIC-2 is present at the plasma membrane and different subcellular compartments, whereas both mutant proteins are mainly seen in the endoplasmic reticulum. Scale bar $=10 \mu \mathrm{m}$. (B) Quantification of colocalisation of wild-type or mutated ClC-2 with endoplasmic reticulum and plasma membrane using Pearson's correlation coefficient, including at least three cells per group. (C) Analysis of wild-type and mutated CIC-2 protein expression in whole lysates and plasma membranes by Western blot show that the expression of mutant CIC-2 protein transiently expressed in COS7 cells is lower than that of the wild-type protein, especially in the plasma membrane. The image shows the result of a representative experiment. Flotillin was used to control and normalise the protein load. (D) Quantification of wild-type and mutant CIC-2 proteins present in whole lysates and in plasma membranes shows that, overall, mutant CIC-2 proteins are not as highly expressed as wild-type CIC-2, suggesting that they are misfolded and unstable. By contrast with the wild-type form, both mutants are retained in the endoplasmic reticulum where they are probably degraded and hardly reach the plasma membrane. The values, obtained from at least three different experiments, were compared with the Mann-Whitney test. * $p<0 \cdot 05$. $\dagger p<0 \cdot 01$. $\neq p<0 \cdot 001$. 
mutated proteins, in particular C-terminally truncated or missense mutants that reach the plasma membrane in substantial amounts, could possibly retain a partial function.

The present study adds to the growing insight into how the brain deals with the continuous shifts of ions and water related to action potentials. Brain white matter mainly consists of myelinated axons and its most important physiological function is impulse conduction. Action potentials are based on shifts in ions, which are obligatorily associated with osmotically driven shifts in water, needing rapid compensation. Any disturbance of the compensatory mechanisms might disrupt impulse transmission or lead to life-threatening changes in brain volume. Brain ion and water homoeostasis is complex and involves electrolytes and organic osmolytes, channels, and transporters, and coordinated processes such as regulatory volume decrease and increase to correct cell volume changes. ${ }^{32,33}$ Astrocytes are central in this process. They are the most abundant and best connected cell type of the so-called panglial syncytium, a vast network of astrocytes, oligodendrocytes, and ependymal cells interconnected by gap junctions. The panglial syncytium is essential for long-distance disposal of ions and water. ${ }^{32,33}$

Action potentials are associated with disposal of submyelinic and intramyelinic potassium. ${ }^{33}$ In myelinated axons, depolarisation is associated with influx of sodium at nodes of Ranvier; compensatory potassium efflux occurs in paranodal regions covered by myelin (appendix). Gap junctions help to transport potassium and osmotic water across myelin layers into astrocytes. Connexin 32 constitutes gap junctions in paranodal myelin; connexin 32 and connexin 47 constitute gap junctions between myelin and astrocytes. The astrocytic syncytium allows rapid dispersion of potassium and buffers the associated volume changes. This process of so-called potassium siphoning prevents action-potential-induced osmotic intramyelinic oedema. ${ }^{33}$ Disruptions of molecular components of these ion and water homoeostatic pathways are associated with intramyelinic oedema in human diseases and mutant mouse models. ${ }^{6,19,33-35}$ No myelin vacuolation occurs in mouse models in the absence of action potentials, ${ }^{6,34}$ showing that hindrance of compensation of action-potential-induced ion and water shifts is the main factor leading to osmotic intramyelinic oedema.

Insight into the mechanisms and molecular components of brain ion and water homoeostatic pathways is still incomplete. Our study provides evidence that $\mathrm{ClC}-2$ is one of these molecular components. The first observation that supports a role of $\mathrm{ClC}-2$ in brain ion and water homoeostasis is the MRI evidence of myelin microvacuolation in patients with CLCN2 mutations. The second observation is the presence of the same MRI abnormalities with evidence of myelin microvacuolation in a patient with a GJB1 mutation. Mutations in GJB1, encoding connexin 32, lead to X-linked Charcot-Marie-
Tooth disease. In addition to peripheral neuropathy, patients with X-linked Charcot-Marie-Tooth disease might have episodes of brain dysfunction, with MRI
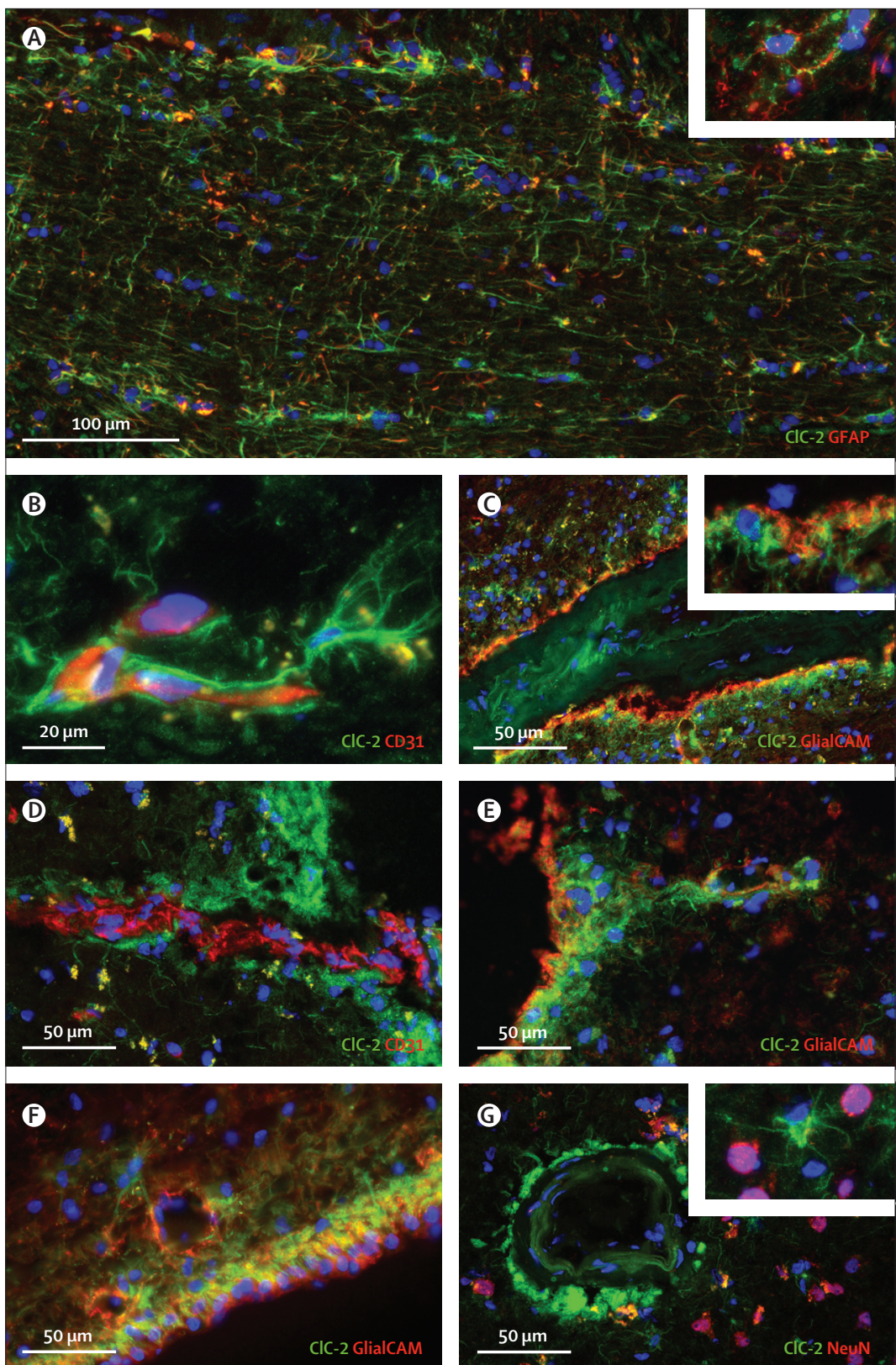

Figure 3: Expression of $\mathrm{ClC}-2$ in a healthy adult human brain

Double immunohistochemical staining of the cerebral white matter for $\mathrm{ClC}-2$ (green) and the astrocyte-specific marker GFAP (red) shows that all GFAP-positive astrocytes express CIC-2 (A). Note the long ClC-2-positive astrocytic processes extending parallel and perpendicular to the direction of the fibre bundle. CIC-2 shows a punctate immunoreactivity, as expected for a membrane protein (inset in A). Colabelling of the same tissue for $\mathrm{ClC}-2$ (green) and the vascular endothelial cell marker CD31 (also known as PECAM1; red) shows that expression of CIC-2 is enhanced in the astrocytes surrounding the blood vessels deep in the parenchyma (B) and the penetrating vessels at the surface of the brain (D). Note the many fine astrocytic processes running along the white matter capillary (B). Perivascular astrocytes (C), subpial astrocytes (E), and ependymal cells (F) coexpress GlialCAM (red) with CIC-2 (green), but GlialCAM

immunoreactivity extends further into the astrocytic processes and endfeet (inset in C). Double staining of the frontal cortex for ClC-2 (green) and the neuronal marker NeuN (red) shows that, in this location, ClC-2 immunoreactivity is restricted to perivascular astrocytes $(\mathrm{G})$. No ClC-2 expression is seen in the NeuN-positive neuronal cells (inset in G). In all images the cell nuclei are stained with DAPI (4;,6-diamidino-2-phenylindole; blue). 

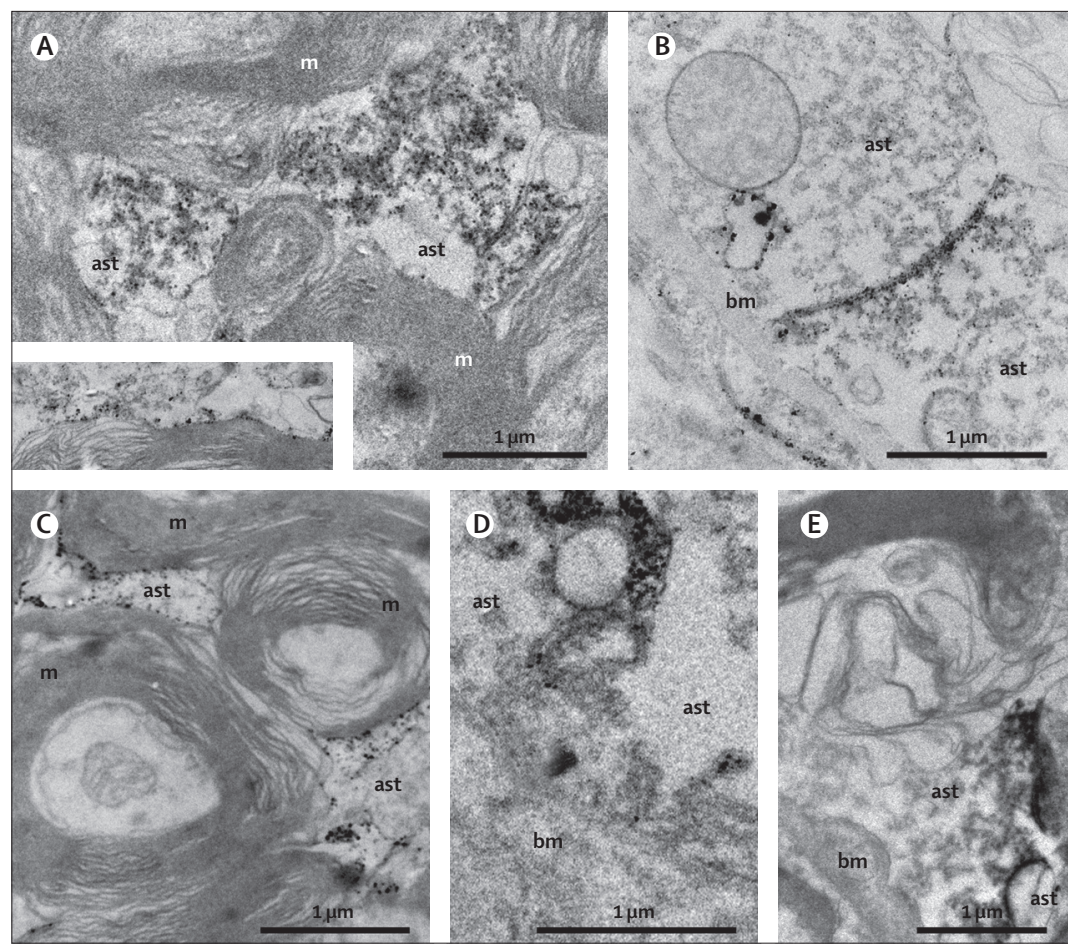

Figure 4: CIC-2, GlialCAM, and MLC1 immunoreactivity in healthy adult white matter

By use of electron microscopy, expression of $\mathrm{CIC}-2$ is detected in astrocytic processes in between myelinated axons (A) and in contact with abaxonal myelin (inset in A). A similar localisation is observed for GlialCAM (C). Intense $\mathrm{ClC}-2$ immunoreactivity is present in astrocytic endfeet surrounding blood vessels (B). Note the enhanced CIC-2 expression in the astrocyte-astrocyte contacts of the endfeet (B). At this site, GlialCAM (D) and MLC1 (E) are also expressed. ast=astrocyte. $\mathrm{bm}=$ basement membrane. $\mathrm{m}=$ myelin.

evidence of transient myelin vacuolation. ${ }^{36}$ We have no explanation for why the mutation detected in the family that we studied has a persistent effect on brain myelin, without any fluctuation, both clinically and on serial MRI. The serious spasticity and cerebellar ataxia probably overshadow the neuropathy, which was only detected by neurophysiological studies after the GJB1 mutation had been identified. Other cases of X-linked Charcot-MarieTooth disease with persistent clinical signs of brain dysfunction and white matter disease on MRI have been reported, but diffusion findings were not mentioned. ${ }^{37}$ The third observation supporting the role of $\mathrm{ClC}-2$ in brain ion and water homoeostasis is the ClC-2 membrane expression in the panglial syncytium, with enhanced expression around blood vessels, in the glia limitans, ependymal lining, and astrocyte-astrocyte contacts in the white matter. The fourth observation is the finding that $\mathrm{ClC}-2$ colocalises with MLC1 and GlialCAM in astrocytic endfeet at the perivascular basal lamina and glia limitans. ${ }^{26,27,38}$ As recently reviewed, ${ }^{22}$ both GLIALCAM and MLC1 mutations cause megalencephalic leukoencephalopathy with subcortical cysts, ${ }^{23,26}$ a disease characterised by myelin vacuolation in the cerebral white matter, in which the corpus callosum, internal capsule, brainstem, and cerebellum are spared in comparison..$^{20,21}$ MLC1 is involved in chloride currents necessary for the astrocytic regulatory volume decrease after cell swelling. ${ }^{39}$ GlialCAM is indispensable for correct localisation of MLC1 and ClC-2. ${ }^{26,27}$ Results of our study suggest that $\mathrm{ClC}-2$ is part of the same ion and water homoeostatic pathways as MLC1 and GlialCAM (appendix).

A limitation of our study is the rather small number of patients. The database on unclassified leukoencephalopathies in Amsterdam contains more than 3000 patients, but only a few patients fulfilled the MRI criteria. It could be that the $\mathrm{ClC}$-2-related leukoencephalopathy is very rare, but we cannot exclude the possibility that the phenotypic variation is much wider and that we have not analysed a sufficient number of patients yet. Another issue is the specificity of the antibody against human ClC-2. To provide full proof, absence of all staining should be shown in brain tissue of a patient with two CLCN2 null mutations. This tissue is not available. However, we did several experiments that support the specificity of the antibody and thus the validity of the immunohistochemical data (appendix).

Many questions remain. The exact role of $\mathrm{ClC}-2$ in brain ion and water homoeostasis is unknown. Why defects in the same pathways cause different white matter disorders is unclear. ClC-2-related and connexin-32related diseases preferentially affect white matter structures that are spared (relatively) by MLC1-related and GlialCAM-related disease. Although recessive mutations in MLC1 and GLIALCAM lead to diffuse cerebral white matter oedema, sparing the corpus callosum, internal capsule, brainstem, and cerebellum, recessive mutations in CLCN2 and GJB1 preferentially cause white matter oedema of the internal capsule, brainstem structures, and cerebellum. Another intriguing question concerns the size of the myelin vacuoles. In MLC, ADC values in the affected white matter are highly increased, ${ }^{40}$ suggesting large vacuoles and increased extracellular spaces, as confirmed by electron microscopy. ${ }^{21}$ In ClC-2-related and connexin-32-related disease, ADC values in affected white matter are low, suggesting small intramyelinic vacuoles and extracellular spaces. Although GlialCAM is supposed to be a chaperone for both MLC1 and ClC-2, ${ }^{26,27}$ recessive GLIALCAM mutations lead to a disease that is indistinguishable from the disease caused by $M L C 1$ mutations and does not share the MRI features of the disease caused by CLCN2 mutations. ${ }^{26}$ We noted that GlialCAM and MLC1 share restricted localisation in distal astrocytic processes, whereas $\mathrm{ClC}-2$ has a more diffuse membrane localisation in astrocytes. These disease observations suggest that GlialCAM is a straightforward chaperone for MLC1, but that its relation with $\mathrm{ClC}-2$ is more complex.

The growing group of known disorders affecting brain ion and water homoeostasis are typically relatively mild compared with other brain disorders, both in mice ${ }^{5,6,19,35}$ and human beings. ${ }^{20,24,26,36}$ The importance of the volume regulation process in the brain is emphasised by the 
Panel: Research in context

\section{Systematic review}

We searched PubMed and Online Mendelian Inheritance in Man with the search terms "leukoencephalopathy", "MLC", "MLC1", "HepaCAM", "GlialCAM", "ClC-2", "CLCN2", "X-linked Charcot Marie Tooth", "connexin 32", and "GJB1", for articles in the English language published between 1969 and Dec 31, 2012. We also searched reference lists of identified papers. We studied reports on epilepsy and leukoencephalopathy related to mutations in the genes CLCN2, MLC1, GLIALCAM, and GJB1, both in mice and humans. Whole-exome sequencing is a well established and effective strategy to identify the gene mutated in several patients with the same disease with autosomal recessive and $\mathrm{X}$-linked recessive inheritance. For interpretation of our molecular genetic findings we used Alamut2.1 (Interactive Biosoftware), 1000 Genomes, and HapMap databases.

\section{Interpretation}

The findings of our study, when added to those of previous work, suggest that there is no evidence for a role of CLCN2 mutations in epilepsy, which should put a definitive end to the discussion. By contrast, following on from findings in rodents, our results show that $\mathrm{ClC}-2$ is involved in brain ion and water homoeostasis in human beings. Defects in MLC1, GlialCAM, connexin 32, and CIC-2 all lead to

leukoencephalopathies characterised by intramyelinic oedema. Observations in X-linked Charcot-Marie-Tooth disease caused by GJB1 mutations, and megalencephalic leukoencephalopathy with subcortical cysts caused by dominant GLIALCAM mutations, show that intramyelinic oedema can be reversible. If means are found to target components of the ion and water homoeostatic pathways, this oedema could be successfully combated. Our study shows that $\mathrm{ClC}-2$ is one of the molecular components, and might be a potential therapeutic target.

observation that it does not rely on just one protein or one pathway. Mutations in one protein only lead to partial and sometimes transient dysfunction. It is important to note that intramyelinic oedema can be reversible. Patients with megalencephalic leukoencephalopathy with subcortical cysts, caused by dominant GLIALCAM mutations, improve or recover spontaneously. ${ }^{24,26}$ In most patients with a GJB1 mutation, the white matter disease is transient. ${ }^{36}$ If means are found to target components of the ion and water homoeostatic pathways, myelin oedema might be successfully combated.

\section{Contributors}

CDe, MB, CDu, NP, CvB, EP, and TEMA did the experiments or analysed the data (or both). CDe, FS, and MSvdK designed and supervised the study. CDe and MSvdK wrote the first draft of the manuscript. DG, VT, ET, FD, AB, CEdD-S, JSV, AV, GU, CY, SGF, VMK, JK, MK, and NIW contributed to patient data or contributed materials essential to the study (or both). All coauthors critically reviewed and approved the last version of the manuscript.

\section{Conflicts of interest}

We declare that we have no conflicts of interest.

\section{Acknowledgments}

We thank patients and families for their participation in the study. We thank the following practitioners for their identification and referral of patients: Janell Kierstein, Johan van Hove, and Abigail Collins from the Children's Hospital of Denver, and Johanna L Schmidt, Children's

National Medical Center, Washington. We thank Raúl Estévez, University of Barcelona, for the anti-MLC1 antibody, and the Dutch Brain Bank for providing part of the tissue used in the study. We thank

James M Powers, University of Rochester, for critical reading of the manuscript. We thank the PIC-PS platform of Centre de Recherche de l'Institut du Cerveau et de la Moelle Epinière (CRICM) and the genotyping and sequencing platform of L'Institut du Cerveau et de la Moelle Épinière for technical assistance; the DNA and cell bank of CRICM for DNA extraction and cell culture; Kees van Rozendaal, Department of Clinical Genomics, Maastricht University Medical Center, for assistance in the exome sequencing of patient 7 and his family; and Petra J W Pouwels, VU University Medical Center, Amsterdam, for her help with the analysis of the apparent diffusion coefficient maps.

\section{References}

1 Planells-Cases R, Jentsch TJ. Chloride channelopathies. Biochim Biophys Acta 2009; 1792: 173-89.

2 Thiemann A, Grunder S, Pusch M, Jentsch TJ. A chloride channel widely expressed in epithelial and non-epithelial cells. Nature 1992; 356: 57-60.

3 Grunder S, Thiemann A, Pusch M, Jentsch TJ. Regions involved in the opening of CIC-2 chloride channel by voltage and cell volume. Nature 1992; 360: 759-62.

4 Jordt SE, Jentsch TJ. Molecular dissection of gating in the ClC-2 chloride channel. EMBO J 1997; 16: 1582-92.

5 Bosl MR, Stein V, Hubner C, et al. Male germ cells and photoreceptors, both dependent on close cell-cell interactions, degenerate upon ClC-2 Cl(-) channel disruption. EMBO J 2001; 20: 1289-99.

6 Blanz J, Schweizer M, Auberson M, et al. Leukoencephalopathy upon disruption of the chloride channel ClC-2. J Neurosci 2007; 27: 6581-89.

7 Sik A, Smith RL, Freund TF. Distribution of chloride channel-2-immunoreactive neuronal and astrocytic processes in the hippocampus. Neuroscience 2000; 101: 51-65.

8 Smith RL, Clayton GH, Wilcox CL, Escudero KW, Staley KJ. Differential expression of an inwardly rectifying chloride conductance in rat brain neurons: a potential mechanism for cell-specific modulation of postsynaptic inhibition. J Neurosci 1995; 15: 4057-67.

9 Rinke I, Artmann J, Stein V. ClC-2 voltage-gated channels constitute part of the background conductance and assist chloride extrusion. J Neurosci 2010; 30: 4776-86

10 Ratte S, Prescott SA. ClC-2 channels regulate neuronal excitability, not intracellular chloride levels. J Neurosci 2011; 31: 15838-43.

11 D'Agostino D, Bertelli M, Gallo S, et al. Mutations and polymorphisms of the CLCN2 gene in idiopathic epilepsy. Neurology 2004; 63: 1500-02.

12 Stogmann E, Lichtner P, Baumgartner C, et al. Mutations in the CLCN2 gene are a rare cause of idiopathic generalized epilepsy syndromes. Neurogenetics 2006; 7: 265-68.

13 Everett K, Chioza B, Aicardi J, et al. Linkage and mutational analysis of CLCN2 in childhood absence epilepsy. Epilepsy Res 2007; 75: 145-53.

14 Saint-Martin C, Gauvain G, Teodorescu G, et al. Two novel CLCN2 mutations accelerating chloride channel deactivation are associated with idiopathic generalized epilepsy. Hum Mutat 2009; 30: 397-405.

15 Combi R, Grioni D, Contri M, et al. Clinical and genetic familial study of a large cohort of Italian children with idiopathic epilepsy. Brain Res Bull 2009; 79: 89-96.

16 Kleefuss-Lie A, Friedl W, Cichon S, et al. CLCN2 variants in idiopathic generalized epilepsy. Nat Genet 2009; 41: 954-55.

17 Haug K, Warnstedt M, Alekov AK, et al. Retraction: Mutations in CLCN2 encoding a voltage-gated chloride channel are associated with idiopathic generalized epilepsies. Nat Genet 2009; 41: 1043.
For the $\mathbf{1 0 0 0}$ Genomes database see http:// www.1000genomes.org For HapMap see http://www hapmap.org 
18 Niemeyer MI, Cid LP, Sepulveda FV, et al. No evidence for a role of CLCN2 variants in idiopathic generalized epilepsy. Nat Genet 2010; 42: 3 .

19 Edwards MM, Marin de Evsikova C, Collin GB, et al. Photoreceptor degeneration, azoospermia, leukoencephalopathy, and abnormal RPE cell function in mice expressing an early stop mutation in CLCN2. Invest Ophthalmol Vis Sci 2010; 51: 3264-72.

20 van der Knaap MS, Barth PG, Stroink H, et al. Leukoencephalopathy with swelling and a discrepantly mild clinical course in eight children. Ann Neurol 1995; 37: 324-34.

21 van der Knaap MS, Barth PG, Vrensen GF, Valk J. Histopathology of an infantile-onset spongiform leukoencephalopathy with a discrepantly mild clinical course. Acta Neuropathol 1996; 92: 206-12.

22 van der Knaap MS, Boor I, Estévez R. Megalencephalic leukoencephalopathy with subcortical cysts: chronic white matter oedema due to a defect in brain ion and water homoeostasis. Lancet Neurol 2012; 11: 973-85.

23 Leegwater PA, Yuan BQ, van der Steen J, et al. Mutations of MLC1 (KIAA0027), encoding a putative membrane protein, cause megalencephalic leukoencephalopathy with subcortical cysts. Am J Hum Genet 2001; 68: 831-38.

24 van der Knaap MS, Lai V, Kohler W, et al. Megalencephalic leukoencephalopathy with cysts without MLC1 defect. Ann Neurol 2010; 67: 834-37.

25 Scheper GC, van Berkel CG, Leisle L, et al. Analysis of CLCN2 as candidate gene for megalencephalic leukoencephalopathy with subcortical cysts. Genet Test Mol Biomarkers 2010; 14: 255-57.

26 Lopez-Hernandez T, Ridder MC, Montolio M, et al. Mutant GlialCAM causes megalencephalic leukoencephalopathy with subcortical cysts, benign familial macrocephaly, and macrocephaly with retardation and autism. Am J Hum Genet 2011; 88: 422-32.

27 Jeworutzki E, Lopez-Hernandez T, Capdevila-Nortes X, et al. GlialCAM, a protein defective in a leukodystrophy, serves as ClC-2 Cl(-) channel auxiliary subunit. Neuron 2012; 73: 951-61.

28 van der Knaap MS, Breiter SN, Naidu S, Hart AA, Valk J. Defining and categorizing leukoencephalopathies of unknown origin: MR imaging approach. Radiology 1999; 213: 121-33.
29 Schiffmann R, van der Knaap MS. Invited article: an MRI-based approach to the diagnosis of white matter disorders. Neurology 2009; 72: 750-59.

30 Dreha-Kulaczewski SF, Brockmann K, Henneke M, et al. Assessment of myelination in hypomyelinating disorders by quantitative MRI. J Magn Reson Imaging 2012; 36: 1329-38.

31 Patay Z. Diffusion-weighted MR imaging in leukodystrophies. Eur Radiol 2005; 15: 2284-303.

32 Benfenati V, Ferroni S. Water transport between CNS compartments: functional and molecular interactions between aquaporins and ion channels. Neuroscience 2010; 168: 926-40.

33 Rash JE. Molecular disruptions of the panglial syncytium block potassium siphoning and axonal saltatory conduction: pertinence to neuromyelitis optica and other demyelinating diseases of the central nervous system. Neuroscience 2010; 168: 982-1008.

34 Neusch C, Rozengurt N, Jacobs RE, Lester HA, Kofuji P. Kir4.1 potassium channel subunit is crucial for oligodendrocyte development and in vivo myelination. J Neurosci 2001; 21: 5429-38.

35 Menichella DM, Majdan M, Awatramani R, et al. Genetic and physiological evidence that oligodendrocyte gap junctions contribute to spatial buffering of potassium released during neuronal activity. J Neurosci 2006; 26: 10984-91.

36 Paulson HL, Garbern JY, Hoban TF, et al. Transient central nervous system white matter abnormality in X-linked Charcot-Marie-Tooth disease. Ann Neurol 2002; 52: 429-34.

37 Siskind C, Feely SM, Bernes S, Shy ME, Garbern JY. Persistent CNS dysfunction in a boy with CMT1X. J Neurol Sci 2009; 279: 109-13.

38 Boor PK, de Groot K, Waisfisz Q, et al. MLC1: a novel protein in distal astroglial processes. J Neuropathol Exp Neurol 2005; 64: 412-19.

39 Ridder MC, Boor I, Lodder JC, et al. Megalencephalic leucoencephalopathy with cysts: defect in chloride currents and cell volume regulation. Brain 2011; 134: 3342-54.

40 van der Voorn JP, Pouwels PJ, Hart AA, et al. Childhood white matter disorders: quantitative MR imaging and spectroscopy. Radiology 2006; 241: 510-17. 